\title{
Helicobacter pylori: diagnosi colturale e ribotipizzazione
}

\author{
Lidia Ricci \\ Laboratorio di Microbiologia,Virologia, Sierologia, A.O. S.M.Nuova, Reggio Emilia
}

Key Words: culture, typeability, Helicobacter pylori.

\section{SUMMARY}

Diagnosis of Helicobacter pylori infection may be established by invasive test. Culture of the organism is an invasive technique and is the most specific method. The test requiring endoscopic biopsy should be performed on the antrum and also at the upper to middle part of the gastric body. Culture can be considered as the reference method and is important if antimicrobial susceptibility testing is required. Sensitivity of the culture is variable and differs among laboratories because of the quality of the organism growth varies with the conditions used. When the culture is performed it is preferable to collect Helicobacter pylori isolates for future studies. Typing systems are used to discriminate between isolates of $H$. pylori for epidemiological and clinical purposes.

Typeability is an important performance criteria of typing systems and the meta-analysis of studies confirmed that PCR- RFLP and RAPD analyses are excellent tecniques. A typeability could be obtained with Automates Ribotyping System when two enzymes, Hae III and Hind III for digestion DNA, are used. Microbiological isolation of Helicobacter pylori is important to determine the resistance status because the failure of therapy is often associated with secondary antibiotic resistance, retreatment should ideally be guided by data on susceptibility.

\section{RELAZIONE}

Helicobacter pylori è la specie appartenente al genere Helicobacter (vedi schema sottostante) che precedentemente era stata attribuita al genere Campylobacter con il nome di Campylobacter pyloridis.

\section{BACTERIA (Regno)<smiles>[CH]=C</smiles>

Il nuovo inquadramento tassonomico del genere Helicobacter si basa sulle conoscenze ottenute dal sequenziamento dei geni della regione $16 \mathrm{~S}$ codificanti per L'RNA ribosomiale ed in figura I si possono osservare le distanze genetiche tra le singole specie. In questo Dendrogramma oltre Helicobacter pyilori è evidenziabile la specie Helicobacter heilmannii in grado come $\mathrm{Hp}$ di colonizzare e causare infiammazione nello stomaco umano. Altre specie quali Helicobacter fennelliae, pullorum, cinaedi, winghanensis, sono state isolate dall'uomo con patologie gastriche o gastroenteriche tuttavia il loro ruolo patogeno è ancora da definire per quanto riguarda il postulato di Koch..

Helicobacter pylori è un battere Gram negativo, che ha una struttura spiraliforme (figura II) e

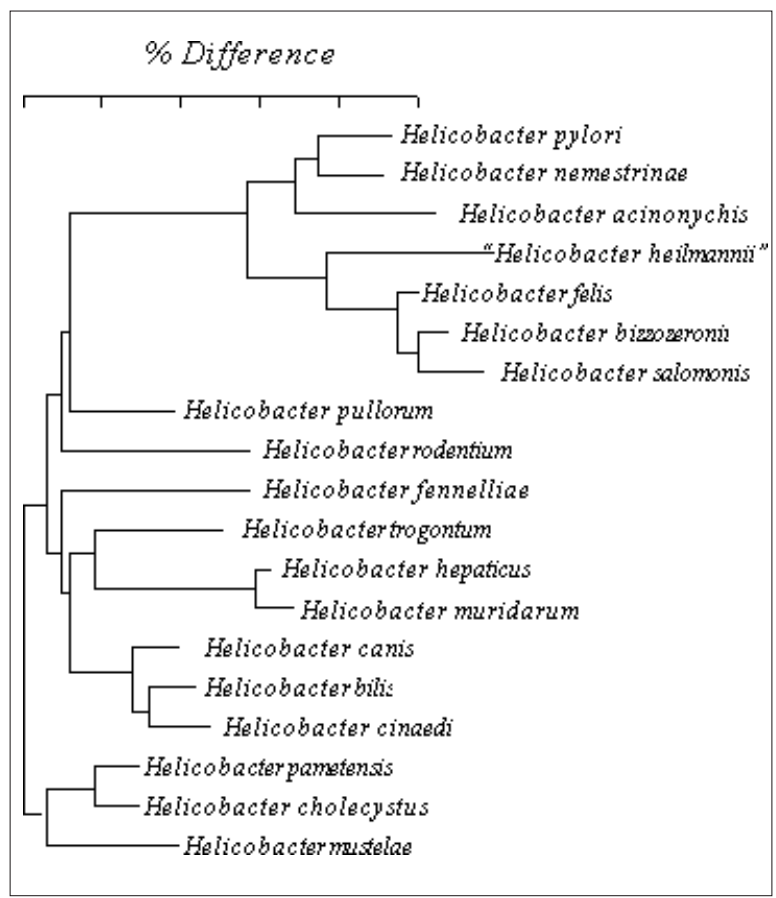

Figura I - Tassonomia del genere Helicobacter dal sequenziamento dei geni I6S RNA (Marshall BJ: Helicobacter pylori in the year 2000)

necessita di un atmosfera microaerofila per la sua crescita in coltura. La sua forma diventa bastoncellare-simile quando è coltivato in agar e nelle colture vecchie sia in terreni liquidi che solidi diventa "coccoide". Secondo le vecchie ipotesi la struttura coccoide rappresenterebbe una modifica sia strutturale che funzionale del germe 


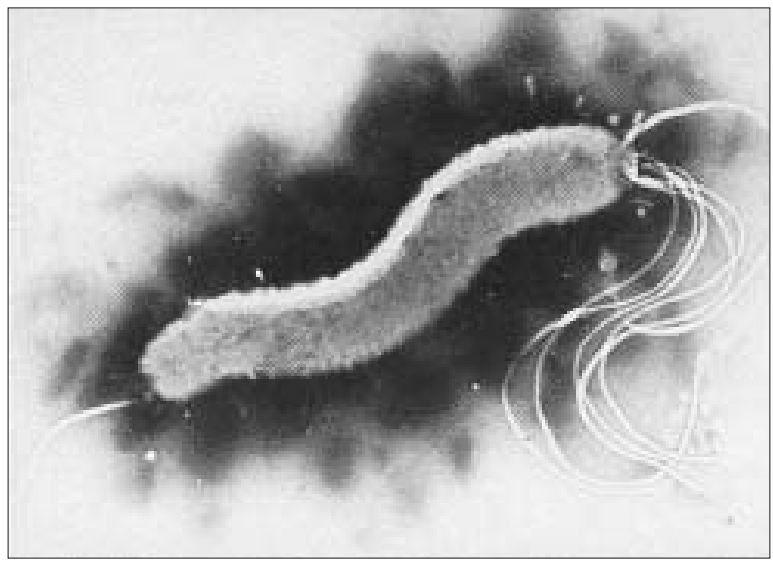

Figura II - Immagine di Helicobacter pylori

in seguito a condizioni sfavorevoli alla sua vitalità (aerobiosi, $\mathrm{pH}$ alcalino,incubazione troppo prolungata dei terreni, temperatura elevata, trattamento con inibitori di pompa dei protoni e con antibiotici ecc.)

Il processo sarebbe reversibile e pertanto il ripristino di condizioni favorevoli allo sviluppo di Hp consentirebbe al germe di riacquistare tutte le sue caratteristiche. Secondo le nuove teorie, presentate anche da Zhukhovisky al congresso internazionale sull'Hp svoltosi ad Atene nel 2002, le forme coccoidi sarebbero dei corpi elementari: sferoblasti e protoplasti con parete cellulare difettosa e rappresenterebbero una fase della riproduzione in vitro del batterio.

Le dimensioni di Hp sono pari a 2,5 - 5,0 $\mu \mathrm{m}$ di lunghezza e $0,5-1,0 \mu \mathrm{m}$ di larghezza.

E' caratterizzato dalla presenza di 3-7 flagelli unipolari che consentono la sua caratteristica motilità.

È rivestito da una membrana glicocalice-simile a struttura lipopolisaccaridica (LPS) a basso potere immunogeno.Metabolizza gli amminoacidi per via fermentativa e quando deve attingere alle sue riserve energetiche utilizza granuli di polifosfato. Ha un genoma che oscilla tra 1,6-1,73 milioni di basi e contiene almeno 2 copie dei geni $16 \mathrm{~S}$ e $23 \mathrm{~S}$ rRNA.

Helicobacter pylori risponde positivamente alle reazioni della ureasi, catalasi ed ossidasi.

È un germe esigente.

Dal 1983, grazie agli scienziati Marshall e Warren, Hp è riconosciuto come agente etiologico di gastrite ed oggi anche di ulcera peptica, carcinoma gastrico ed altre patologie.

Da allora si è rivolta una particolare attenzione al battere e sono stati prodotti numerosi lavori scientifici che hanno toccato vari aspetti, compreso quello della diagnosi di laboratorio

In tabella 1 sono illustrati i metodi diagnostici di laboratorio secondo la suddivisione di Cutler del
1995 tra metodi invasivi e non invasivi.

La differenziazione si basa sul prelievo del materiale da esaminare, che per i metodi invasivi richiede la Esofagogastroduodenoscopia (EGDS). I metodi non invasivi sono invece eseguiti su vari tipi di materiali più facilmente reperibili.

\section{Tabella I- Metodi diagnostici di laboratorio.}

\begin{tabular}{|ll|}
\hline \multicolumn{2}{|c|}{ DIAGNOSI DI LABORATORIO DI HELICOBACTER PYLORI } \\
\hline METODI INVASIVI & METODI NON INVASIVI \\
\hline Coltura & Urea breath test \\
\hline Istologia & Antigene fecale \\
\hline Ureasi test (test rapido) & Sierologia \\
\hline Microscopia (test rapido) & Anticorpi urinari \\
\hline PCR ed altre tecniche & $\begin{array}{l}\text { PCR ed altre tecniche di } \\
\text { di biologia molecolare }\end{array}$ \\
& $\begin{array}{l}\text { (da saliva,feci,placca } \\
\text { dentaria,succogastrico ecc.) }\end{array}$ \\
\end{tabular}

La coltura di Helicobacter ylori rappresenta il gold standard nella diagnostica di laboratorio pertanto la sua specificità è pari al $100 \%$, molto variabile è invece il valore della sensibilità.

In tabella 2 sono messi a confronto i suoi vantaggi con gli svantaggi in base alle conoscenze attuali.

Tabella 2 - Confronto dei vantaggi e svantaggi della coltura di Hp

\begin{tabular}{|ll|}
\hline \multicolumn{2}{|c|}{ COLTURA DI HELICOBACTER PYLORI } \\
\hline VANTAGGI & SVANTAGGI \\
\hline Gold Standard & Tecnica Invasiva \\
\hline Specificità I00\% & $\begin{array}{l}\text { Tempi di esecuzione e } \\
\text { Sensibilità variabile (80-99\%) }\end{array}$ \\
\hline $\begin{array}{l}\text { Tipizzazione dei ceppi e } \\
\text { studio della patogenesi }\end{array}$ & Costi elevati \\
\hline $\begin{array}{l}\text { Determinazione } \\
\text { delle resistenze }\end{array}$ & $\begin{array}{l}\text { Accuratezza variabile } \\
\text { (agar diluizione, } \\
\text { agar diffusione, E-test) }\end{array}$ \\
\hline
\end{tabular}

I valori di sensibità della coltura riferiti da vari autori sono illustrati nelle tabelle 3,4,5 e sono messi a confronto con altre tecniche diagnostiche.

Tabella 3 - Sensibilità della coltura

$$
\text { COLTURA DI HELICOBACTER PYLORI }
$$$$
\text { VALUTAZIONE DELLA SENSIBILITÀ }
$$

Bayerdorffer et al. 1989: 95\% con due biopsie

Mègraud et al. 1993: 94\%vs 89\% PCR

Lindt et al. 1997: 97\% su UBT

Mègraud et al. 1999: 99\% su UBT 
Tabella 4 - Sensibilità della coltura

COLTURA H. PYLORI
\begin{tabular}{|l|l|l|l|}
\hline \multicolumn{4}{|c|}{ VALUTAZIONI DELLA SENSIBILITÀ } \\
\hline Logan R., Walker M.Mr et al. ( BMJ) 2001: 80-90\% \\
\hline Test invasivi & Sensibilità & Specificità & Eseguibilità \\
Istologia & $88-95 \%$ & $90-95 \%$ & ++++ \\
Coltura & $80-90 \%$ & $95-100 \%$ & ++ \\
Test Ureasi & $90-95 \%$ & $90-95 \%$ & ++++ \\
& & & \\
\hline Test non invasivi & Sensibilità & Specificità & Eseguibilità \\
13 C-UBT & $90-95 \%$ & $90-95 \%$ & ++++ \\
14C-UBT & $86-95 \%$ & $86-95 \%$ & +++ \\
Elisa & $80-95 \%$ & $80-95 \%$ & +++ \\
NPT & $90-95 \%$ & $70-85 \%$ & ++++ \\
Antigene fecale & $90-95 \%$ & $90-95 \%$ & ++ \\
& & & \\
\hline
\end{tabular}

Tabella 5 - Sensibilità della coltura

\begin{tabular}{|} 
COLTURA H. PYLORI \\
VALUTAZIONI DELLA SENSIBILITÀ \\
\begin{tabular}{|l|l|l|l|l|}
\hline \multicolumn{3}{|l|}{ Kisa O. et al. Diag.Mic.and Infectious Disease 2002: $80 \%$} \\
\hline Test & Sensibilità & Specificità & VPP & VPN \\
\hline Nested PCR & $98 \%$ & $100 \%$ & $100 \%$ & $98 \%$ \\
PCR & $86 \%$ & $100 \%$ & $100 \%$ & $91 \%$ \\
Coltura & $80 \%$ & $100 \%$ & $100 \%$ & $87 \%$ \\
Istologia & $77 \%$ & $97 \%$ & $94 \%$ & $85 \%$ \\
Giemsa & $52 \%$ & $97 \%$ & $92 \%$ & $73 \%$ \\
\hline
\end{tabular}
\end{tabular}

Dalla tabella 4 si può osservare che Logan $\mathrm{R}$ nel suo studio descrive un alto valore di specificità per la coltura, vicino al 100\%, ma la sensibilità riscontrata è più bassa degli altri test, sia invasivi che non invasivi,confrontati.Dalla sua indagine la determinazione dell'Urea Breath Test con l'impiego di ${ }^{13} \mathrm{C}$ mostra i più alti valori di sensibilità ed un valore di specificità che poco si discosta da quello della coltura: $90-95 \%$ vs $95-100 \%$

In Tabella 5 le tecniche di biologia molecolare ed in particolare la Nested PCR mostrano i migliori risultati ai fini della diagnostica che utilizza un prelievo bioptico.

L'accuratezza della coltura è importante per migliorare la sua sensibilità.

In tabella 6 ne sono schematizzate le fasi operative.

Come per tutte le indagini microbiologiche un prelievo corretto è essenziale per la coltura ed è abitualmente eseguito dal gastroenterologo con l'Esofagastroduodenoscopia (figura III).

Nel 1995 è stato pubblicato da Perez-Trallero. su Lancet un metodo di raccolta meno invasivo: Sring-test o Entero-test Hp descritto anche da altri autori. Consiste nell'utilizzo di una fibra di nylon di $90 \mathrm{~cm}$ di lunghezza contenuta in una capsula di gelatina di $2-5 \mathrm{~cm}$ di diametro.

I primi $30 \mathrm{~cm}$ di questa fibra non sono assorbenti contrariamente agli altri $60 \mathrm{~cm}$.

Il paziente su cui si deve eseguire il prelievo deve essere digiuno dalla notte, gli viene fatta ingerire la capsula tenendo fuori i primi $30 \mathrm{~cm}$ del filo

Tabella 6 - Caratteristiche colturali

\begin{tabular}{|c|c|c|c|c|c|}
\hline \multicolumn{6}{|c|}{ COLTURA HELICOBACTER PYLORI - SCHEMA OPERATIVO } \\
\hline PRELIEVO & TERRENO & $\begin{array}{l}\text { TEMPERATURA } \\
\text { INCUBAZIONE }\end{array}$ & $\begin{array}{c}\text { TEMPO } \\
\text { INCUBAZIONE }\end{array}$ & $\begin{array}{l}\text { ATMOSFERA } \\
\text { INCUBAZIONE }\end{array}$ & $\begin{array}{c}\text { CEPPO } \\
\text { CONTROLLO }\end{array}$ \\
\hline \multirow{2}{*}{$\begin{array}{l}\text { Biopsie gastriche } \\
\text { multiple } \\
\text { (antro+corpo) } \\
\text { EGDS } \\
\text { O } \\
\text { STRING-TEST }\end{array}$} & $\begin{array}{c}\text { H.pylori } \\
\text { Selective agar } \\
0 \\
\text { Pylori agar }\end{array}$ & $35-37^{\circ} \mathrm{C}$ & 3-7 GIORNI & Microaerofila & $\begin{array}{l}\text { ATCC } \\
43504\end{array}$ \\
\hline & $\begin{array}{c}\text { Blood agar } \\
\text { Brain heart } \\
\text { infusion, }(\mathrm{BHI}) \\
\text { Brucella, } \\
\text { Skirrow's } \\
+ \\
\text { horse bood o } \\
\text { horse serum o } \\
\text { sheep blood }\end{array}$ & $35-37^{\circ} \mathrm{C}$ & 3-7 GIORNI & Microaerofila & $\begin{array}{l}\text { ATCC } \\
43504\end{array}$ \\
\hline
\end{tabular}


con l'ausilio di $100 \mathrm{ml}$ di $\mathrm{H}_{2} 0$. Dopo un ora di totale rilassamento del paziente si procede al ritiro della fibra da cui vengono scartati i primi 30 $\mathrm{cm}$ perchè contaminati dalla flora orale e nasofaringea.

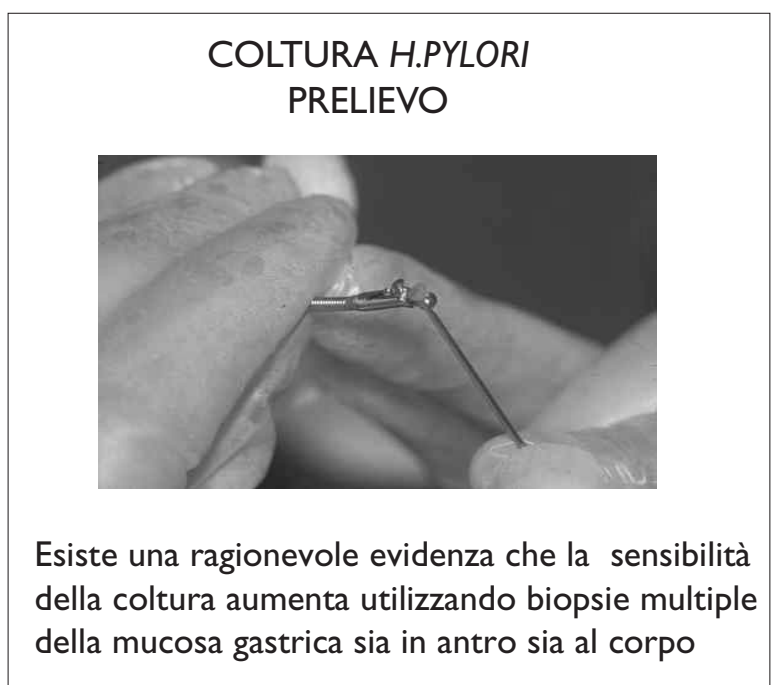

Figura III - Biopsia della mucosa gastrica

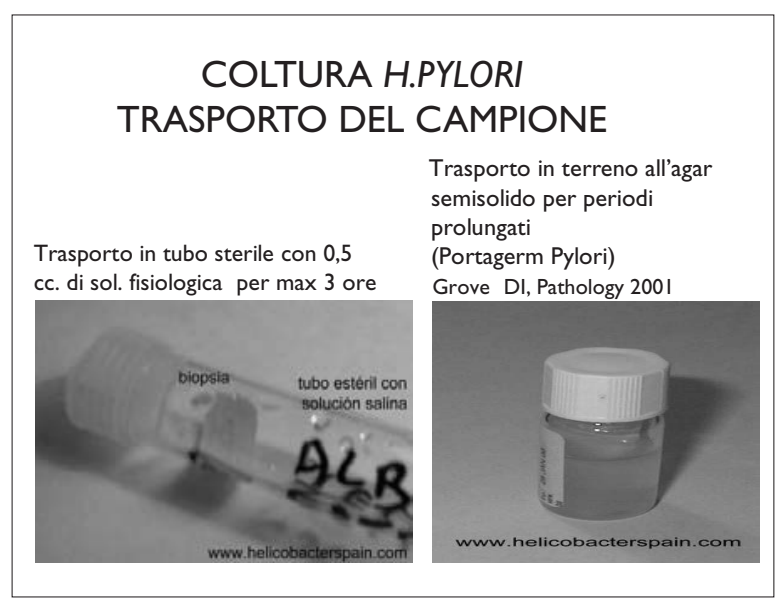

Figura IV - Modalità di trasporto del campione

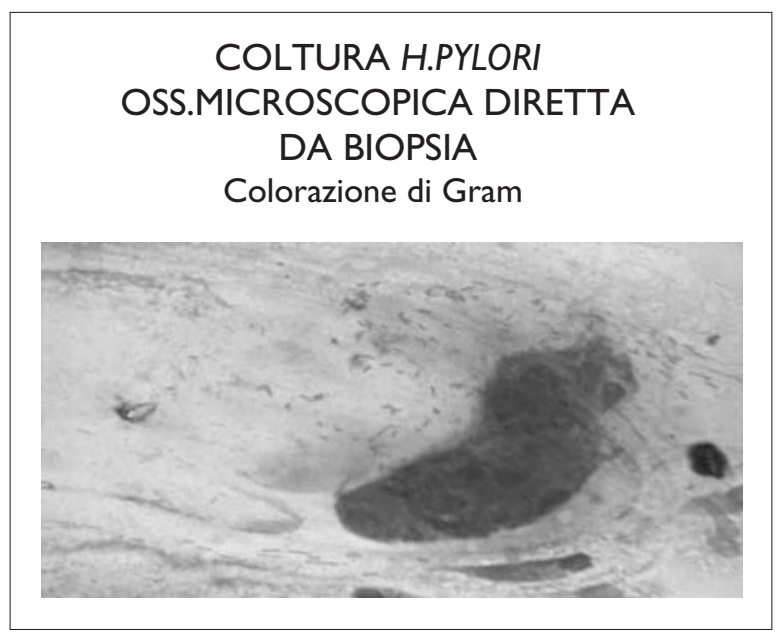

Figura V - Controllo microscopico
Dei rimanenti $60 \mathrm{~cm}, 2 \mathrm{~cm}$ vengono utilizzati per il test dell'ureasi ed il resto viene diviso in 2 frammenti utilizzati per l'allestimento colturale.

Attualmente non ci sono studi sufficienti di confronto fra questa tecnica di prelievo e quella da EGDS.

Dopo il prelievo il materiale deve essere consegnato al laboratorio e la scelta del mezzo di trasporto è condizionata dal tempo impiegato fino all'allestimento della coltura. Per un tempo massimo di 3 ore ogni singola biopsia può essere posta in provetta sterile contenente $0,5 \mathrm{cc}$ di soluzione fisiologica. Per periodi di tempo più lunghi è consigliabile utilizzare un contenitore del commercio come Portagerm pylori che contiene una miscela di peptoni ed antibiotici. Grove ha pubblicato nel 2001 su Pathology uno studio di comparazione tra vari metodi di trasporto e conclude che l'utilizzo di questo mezzo garantisce la miglior sopravvivenza del germe (figura IV)

Dal materiale prelevato si deve procedere all'allestimento di un preparato microscopico colorato secondo Gram, la cui osservazione (figura V) può consentire di dare in breve tempo informazioni al clinico specie quando sono presenti batteri gram negativi con spiccata morfologia elicoidale. La sensibilità del metodo microscopico è condizionata da:

1) esperienza dell'operatore

2) qualità e sede di prelievo

$3)$ terapia con antibiotici ed antisecretori

4) scarsa densità batterica.

Una piccola porzione del materiale bioptico viene utilizzato anche per il test dell'ureasi illustrato in figura VI. In una provetta contenente urea e l'indicatore di $\mathrm{pH}$ ( rosso-fenolo) si pone il materiale. Se è presente Helicobacter pylori l'enzima ureasi presente sulla superficie del battere vitale scinde l'urea in bicarbonato ed ammoniaca determinando una variazione di $\mathrm{pH}$ da acido ad alcalino che conferisce al brodo una variazione di colore da giallino a violaceo indice di attività ureasica.

Tale test rapido (2-5 ore, $\max 24$ ) ha una sensibilità pari al $90-95 \%$ tuttavia presenta dei limiti dovuti a:

1) falsi positivi per presenza di altri batteri ureasi produttori come Proteus SP., Klebsiella SP. ecc.

2) falsi negativi per cariche batteriche molto basse

3) falsi negativi in caso di presenza di tracce di sangue,farmaci o antisecretori.

La semina del campione deve essere eseguita contemporaneamente su un terreno selettivo ed uno non selettivo. Tale raccomandazione consentirebbe di ottenere la massima sensibilità colturale. 
Nel nostro laboratorio allestiamo una piastra di Pyori agar come terreno selettivo ed una piastra di Trypticase soy agar al 5\% di sangue di montone come terreno non selettivo. Qualunque sia il terreno scelto per la coltura è indispensabile tener conto che Helicobacter pylori è un germe esigente che richiede tempi lunghi e facile è la contaminazione soprattutto da funghi.

In tabella 7 sono indicati i supplementi che favoriscono la coltura. Si può osservare che antibiotici utili sono vancomicina e trimethoprim ed amphotericina è l'antimicotico più utilizzato. L'Helicobacter pylori è sensibile all'attività di clindamicina, cephalosporine, colistina e polimixina $\mathrm{B}$ e pertanto non debbono essere presenti nella composizione dei terreni (inibirebbero la crescita di HP).

L'incubazione dei terreni deve essere effettuata secondo le caratteristiche illustrate nello schema sottostante (figuraVII):

L'identificazione di Hp rappresenta la fase successiva alla sua replicazione sui terreni di coltura e si basa su:

1) aspetto delle colonie

2) osservazione microscopica

3) valutazione dell'attività enzimatica di $\mathrm{Hp}$ vitali

Le colonie di Hp sono piccole, brillanti e trasparenti sia sul terreno selettivo che in agar sangue come illustrato in figura VIII e IX.

L'osservazione microscopica di una sospensione in fisiologica delle colonie consente di verificare la gram- negatività e l'aspetto simil-bastoncellare conferito dalla coltura in terreno agarizzato (figura X).

Le reazioni che valutano l'attività enzimatica sono elencate nella figura XI

a)l'ureasi descritta precedentemente (figura VI ) come test rapido è utilizzata anche per l'identificazione quando si usano le colonie invece che la biopsia. L'ureasi è è una metallo-proteina(nichel) formata da due sub unità e costituisce il $5-10 \%$ delle proteine totali del battere.

b)la catalasi è un enzima che determina la seguente reazione: $2 \mathrm{H}_{2} \mathrm{O}_{2} \Rightarrow 2 \mathrm{H}_{2} \mathrm{O}+\mathrm{O}_{2}$

c) ossidasi è l'enzima capace di ossidare un determinato substrato in presenza di ossigeno e la reazione è visibile per la formazione di un composto

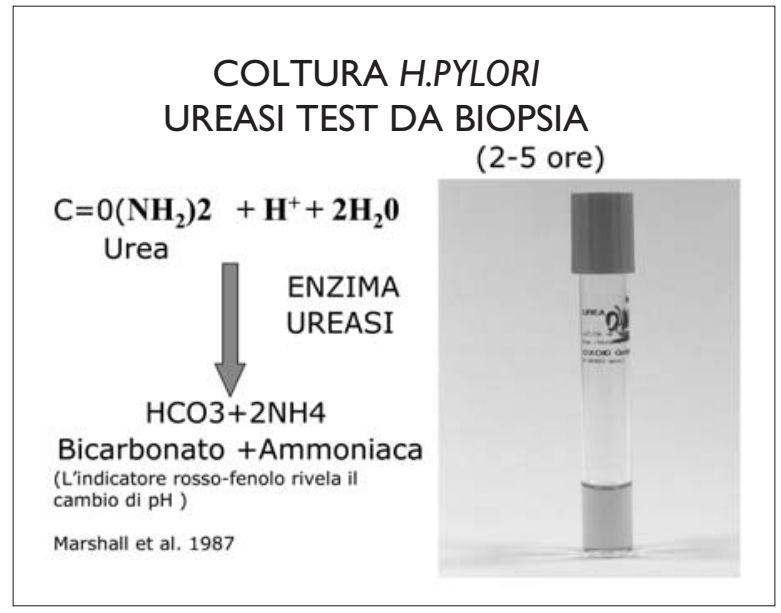

Figura VI - Ureasi Test

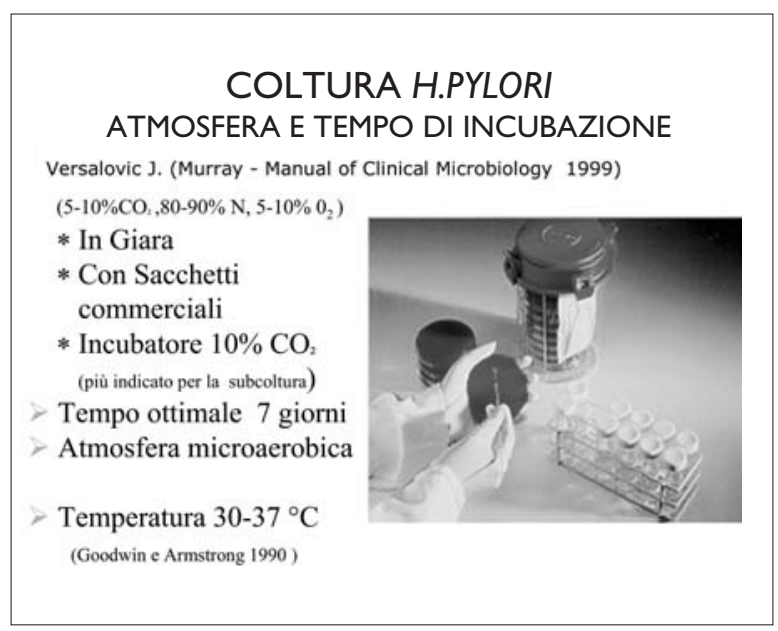

Figura VII - Caratteristiche di incubazione dei terreni

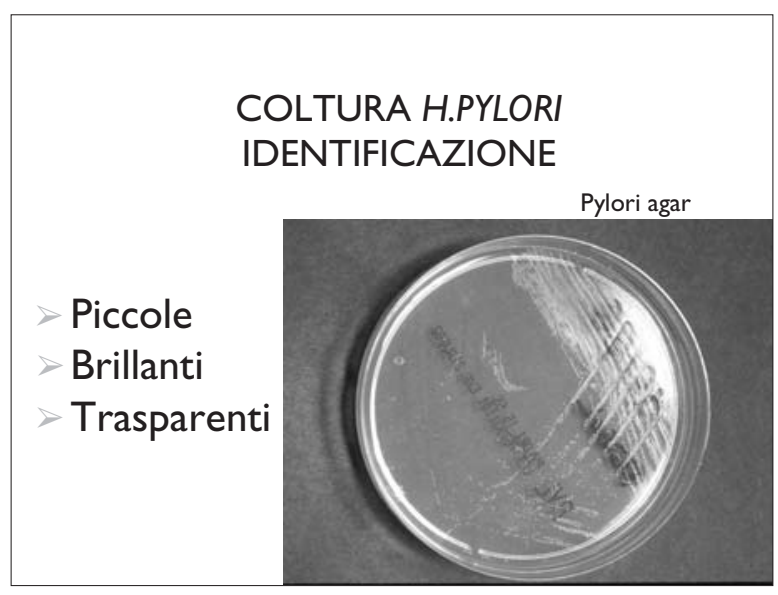

Figura VIII - Aspetto delle colonie su Pylori agar

Tabella 7 - Caratteristiche dei terreni

\begin{tabular}{|l|l|l|}
\hline \multicolumn{2}{|c|}{$\begin{array}{c}\text { COLTURA DI HELICOBACTER PYLORI } \\
\text { CARATTERISTICHE DEI TERRENI }\end{array}$} \\
\hline SELETTIVI E NON SELETTIVI & SUPPLEMENTI UTILI & SUPPLEMENTI DANNOSI \\
\hline Arricchimento con sangue o siero & Vancomicina & Clindamicina \\
bovino,carbone, emulsione d'uovo, & Amphotericina & Cephalosporine \\
ciclodestrine, colesterolo ecc. & Trimethoprim & Colistina o Polimixina B \\
\hline
\end{tabular}


colorato(porpora).

Per l'identificazione basata sulle prove biochimiche ottimi risultati si ottengono anche con l'utilizzo di gallerie commerciali come api Campy (Biomerieux).

Una volta isolato il ceppo di Helicobacter può essere conservato per un tempo anche di 6 mesi in congelatore a $-70^{\circ} \mathrm{C}$ in brodo contenente il 20$25 \%$ di glicerina o in microbank commerciali.

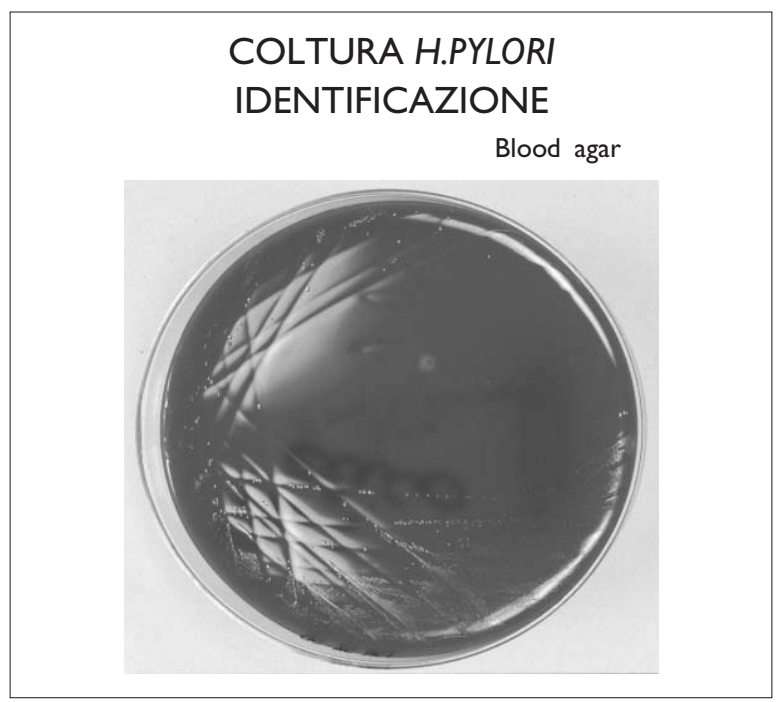

Figura IX

Aspetto delle colonie su Trypticase Soy agar al 5\%

\section{COLTURA H.PYLORI IDENTIFICAZIONE}

Osservazione microscopica del preparato colorato secondo Gram

(forma bastoncellare-simile) (Godwin et al. 1987)

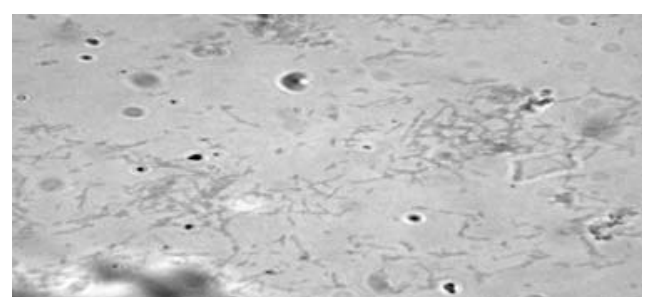

Figura X - Microscopia di Hpda coltura

\section{COLTURA H.PYLORI IDENTIFICAZIONE}

Espressione dell'attività enzimatica di superficie di H.pylori vitali.

$>$ ureasi (Bode et al.1993)

$>$ catalasi (Phadnis et al. 1996)

> superossido dismutasi (SOD)

(Spiegelhalder et al. 1993)

Figura XI - Reazioni di identificazione di $H p$
L'indagine colturale richiede un certo impegno organizzativo ed economico e non incrementa significativamente la sensibilità diagnostica, inoltre eseguita subito dopo terapia eradicante può dare risultati falsamente negativi.

In agosto del 1997 è stata pubblicata la prima sequenza completa del genoma di Helicobacter pylori 26695 (Tomb t al. Nature 1997;388:539547) rappresentata in figura XII.

Nel 1999 (figura XIII) è stato sequenziato un secondo genoma di H.pylori J99 (Alme t al. Nature 1999; 397:176-180)

Nel genoma rappresentato nella sua struttura circolare ciascun colore rappresenta un diverso gruppo funzionale di geni. Hp è caratterizzato da una grande variabilità genetica di cui non si conoscono bene le basi molecolari.Gruppi clonali di H.pylori sono stati riscontrati in gruppi etnici diversi in Cina, Nuova Zelanda ed altri paesi. Wang ed altri puntualizzano che le mutazioni sono il fattore più importante nell'indurre le variazioni genetiche di Hp. La conoscenza del DNA di Hp consente di utilizzare le tecniche di biologia molecolare per:

1) Studio dei possibili fattori di patogenicità

2) Studio delle mutazioni nei geni che codificano per la resistenza agli antibiotici

3) Individuazione delle infezioni miste dovute a differenti ceppi di Hp presenti nel medesimo paziente.

4) Identificazione, Caratterizzazione e Tipizzazione degli isolati di Helicobacter pylori.

La tipizzazione di Hp è interessante sul piano epidemiologico e clinico ed il potere discriminatorio e la tipizzabilità sono importanti fattori per la scelta delle tecniche oggi disponibili.

Da una metaanalisi di Cristophe Burucoa ed altri pubblicata nel 1999 sul Journal of Clinical Microbiology la tecnica: PCR-based restriction fragment lenght polymorphism analysis (PCRRFLP) garantisce la miglior tipizzabilità. Esistono comunque altre tecniche gia disponibili e altre in via di definizione. L'accuratezza diagnostica delle tecniche molecolari dovrà essere oggetto di revisioni sistematiche di tutti i lavori scientifici prodotti al fine di una scelta basata sulla miglior evidenza disponibile.

La ribotipizzazione è una tecnologia tra le varie esistenti che ha il vantaggio di poter essere eseguita anche in automazione (Riboprinter, Oxoid). La ribotipizzazione è applicabile solo a grandi quantità di DNA e pertanto necessita di una coltura del microrganismo o di una grande quantità di nucleotidi. In figura XIV sono elencate le caratteristiche della metodologia.

Il primo caso di evidenza di trasmissione dell'infezione da $\mathrm{Hp}$ tra madre-bambino è stato rivelato 
tramite tecnica di Ribotipizzazione o Ribotyping e nella figura XV è schematizzato lo studio di Taneike Ikue ed altri pubblicato su Clinical and Diagnostic Laboratory Immunology nel 2001.

Il caso riguarda una famiglia giapponese costituita da padre, madre e tre bambini rispettivamente di 9,7 e 4 anni.Il bambino di 9 anni (numero 1) ed il padre erano infetti con lo stesso ceppo di $\mathrm{Hp}$ manifestando ulcera duodenale. Il bambino di 7 anni (numero 2) sintomatico per ulcera duodenale e la madre asintomatica erano infetti con uno stesso tipo di H.pylori diverso però da quello del padre e del bambino di 9 anni. Solo il bambino di 4 anni non era infetto e non mostrava alcuna patologia da Hp. Il padre ed i due bambini con ulcera venivano sottoposti a terapia, l'eradicazione veniva confermata da un controllo eseguito dopo 7 settimane dalla fine del trattamento.Tuttavia 36 settimane più tardi il bambino di 9 anni $\left(n^{\circ} 1\right)$ mostra reinfezione con lo stesso ceppo della madre che era l'unica positiva per Hp e non sottoposta a terapia.

La Ribotipizzazione può essere automatizzata con sistema Riboprinter e pertanto le fasi operative illustrate nelle figure XVI e XVII sono eseguite in automazione.La fase di preparazione precedente al carico del campione sullo strumento consiste nel prelievo di colonie di $\mathrm{Hp}$ dal terreno di coltu$\mathrm{ra}$, disattivazione del germe tramite trattamento ad alta temperatura $\left(90-95{ }^{\circ} \mathrm{C}\right)$ ed aggiunta di enzimi litici dopo raffreddamento. L'utilizzo di due enzimi di restrizione Hae III e Hind III per la digestione del DNA di Helicobacter pylori aumenta il potere discriminatorio della tecnica di Ribotipizzazione e pertanto una maggiore differenziazione tra gli isolati in esame.

In figura XVIII è illustrato il pattern genetico di 8 isolati di Helicobacter pylori ottenuto con Ribotipizzazione automatica. Solo 2 profili sono uguali e pertanto gli isolati appartengono allo stesso ribotipo, gli altri sei sono tutti diversi fra loro. Tale dato può fornire informazioni importanti sia sul piano epidemiologico per conoscere meglio le vie di trasmissione, le aree di diffusione di un ceppo piuttosto che un altro e sul piano clinico perchè ribotipi diversi potrebbero manifestare una diversa patogenicità.

La coltura è pertanto un mezzo diagnostico che non può essere ignorato dal clinico e credo che il suo valore sia indicato in questa frase tratta dalle linee guida Giapponesi del 2001 di Masahiro Asaka ed altri:

If repeat eradication therapy is being considered, culture and susceptibility testing is recommended.

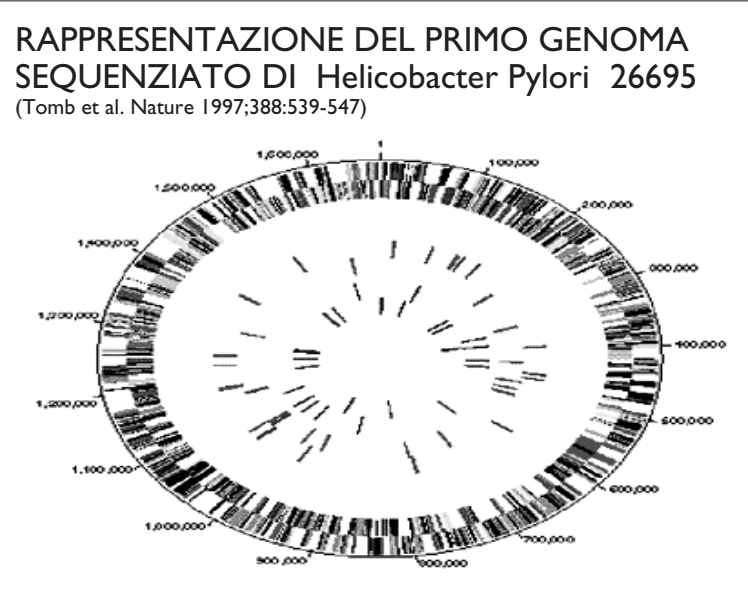

Figura XII - Genoma di H.pylori 26695

RAPPRESENTAZIONE DEL SECONDO GENOMA DI H.pylori $J 99$ SEQUENZIATO (Alm et al. Nature 1999)

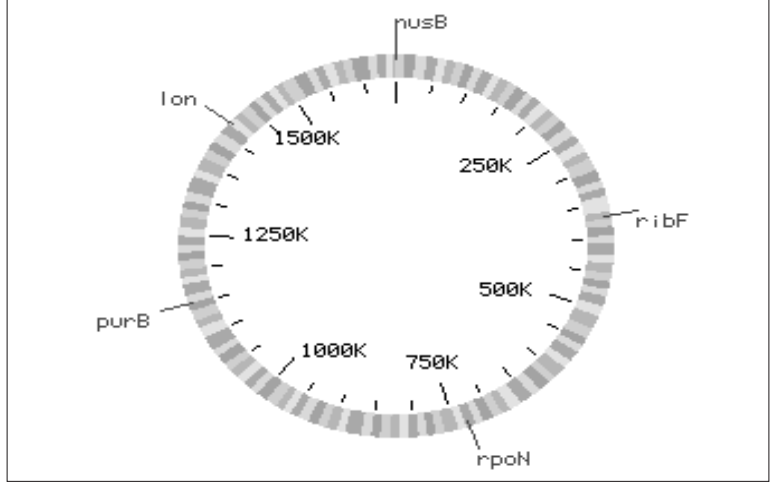

Figura XIII - Genoma di H.pylori J99

\section{RIBOTIPIZZAZIONE}

Consente sequenziamento e confronto dei geni codificanti gli RNA ribosomici (regione 16S 23S) ed rDNA è presente in tutte le specie batteriche con:

regioni altamente conservate (strutturali e funzionali), che consentono la discriminazione di specie

regioni variabili, sulle quali si basa la differenziazione oltre il livello di specie Hubner et al. 1995; Bruce et al 1995

Figura XIV - Ribotipizzazione

TIPIZZAZIONE DI CEPPI DI HELICOBACTER PYLORI IN UNA FAMIGLIA GIAPPONESE CON RIBOTYPING

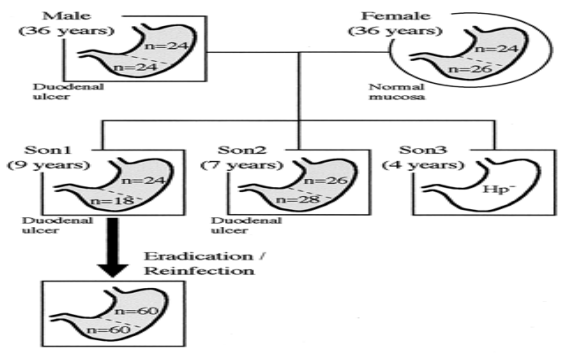

Figura XV - Evidenza di trasmissione intrafamiliare di $H p$ 


\section{RIBOTIPIZZAZIONE AUTOMATICA}

\section{FASI OPERATIVE}

Estrazione DNA cromosomico

Digestione DNA con un enzima di restrizione (Hae III e/o Hind III )

Elettroforesi in gel di agarosio

Southern blotting

Ibridazione con sonde costituite da rRNA o rDNA marcato.

Figura XVI - Fasi della Ribotipizzazione

\section{RIBOTIPIZZAZIONE AUTOMATICA}

\section{FASI OPERATIVE}

Reazioni chimiche/enzimatiche sulla membrana

Chemioluminescenza dei frammenti

Cattura dell'immagine con video camera digitale

Computerizzazione dell'immagine di Ribopattern

Figura XVII - Fasi della Ribotipizzazione

\section{CARATTERIZZAZIONE IN RIBOTIPI (IMPRONTA GENETICA O PATTERN) DI HELICOBACTER PYLORI}

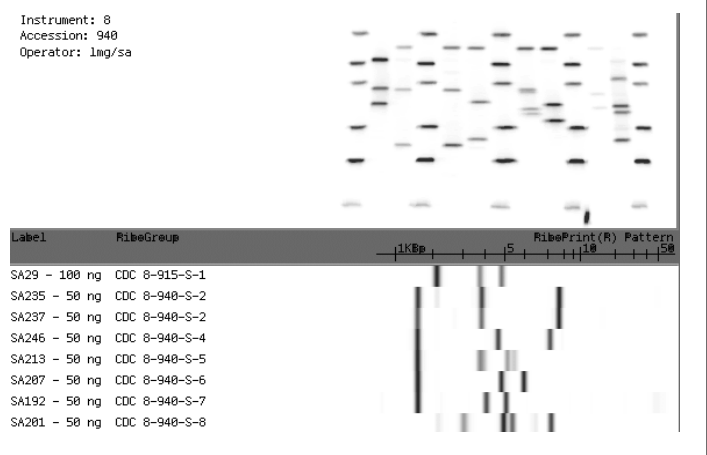

Figura XVIII - Pattern di 8 ribotipi di HP

\section{BIBLIOGRAFIA}

1. Alm RA, Ling LS, Moir DT, King BL, Brown ED, Doig PC et al.-Genomic-sequence comparison of two unrelated isolates of the human gastric pathogen Helicobacter pylori-Nature 1999;397:176-180

2. Amy L Samuels, Helen M Windsor, Grace YHo, Luke D. Goodwin, and Barry J. Marshall-Culture of Helicobacter pylori from a Gastric String May Be an Alternative to Endoscopic Biopsy-.

J Clin Microb, 2000;38(6):2438-2439

3. Anthony P Roberts, Susan M Childs, Greg Rubin,
Niek J de Wit-Tests for Helicobacter pylori infection: a critical appraisal from primary car-Family Practice.Oxford University Press 2000.

4. Bayerdorffer E, Oertel H, Lehn N, Kasper G, Mannes GA, Sauerbru Stolte M-Topographic association between active gastritis and Campylobacter pylori colonisation - J Clin Pathol 1989; 42(8):834-9

5. Bamford KB, Bickley J, Collins JS, Johnston BT, Potts S, Boston V,Owen RJ,Sloan JM-.

Helicobacter pylori: comparison of DNA fingerprints provides evidence for intrafamilial infection. Gut1993; 34(10):1348-1350.

6. Barry J Marshall-Helicobacter pylori in the year 2000

7. Bode G, Mauch F, Malfertheiner P-The coccoid forms of Helicobacter pylori. Criteria for their viabilityEpidemiol Infect 1993;111:483-490.

8. CDC, 2000-History of Ulcer Diagnosis and Treatment.

9. CeVEAS

Linea guida sulla diagnostica dell'Helicobacter pylori-Centro per la Valutazione della Efficacia della Assistenza Sanitaria (2000).

10. CF Weijnen, N.J.De Wit, MENumans, E J Kuipers, et al - Helicobacter pylori testing in the primary care setting: which diagnostic test should be used?Alimentary Pharmacology \& Therapeutics 2001; 15 (8) 1205.

11. Christophe Burucoa,Vincent Lhomme, Jean Louis Fauchere - Performance Criteria of DNA Fingerprinting Methods for Typing of Helicobacter pylori Isolates:Experimental Results and MetaAnalysis.-J Clin Microb1999,37(.12):4071-4080

12. Chey WD, Fendrick AM.-Non invasive Helicobacter pylori testing for the "test-and-treat" strategy: a decision analysis to assess the effect of past infection on test choice-

Arch Intern Med 2001;161(17):2129-32

13. Chung IK, Hong SJ, Kim EJ, Cho JY, Kim HS, Park $\mathrm{SH}$, et al.-What is the best method to diagnose Helicobacter infection in bleeding peptic ulcers:a prospective trial-. Korean J Intern Med 2001;16(3):147-52

14. Cutler AF, Havsta S, Ma CK, et al.-Accuracy of invasive and non invasive test to diagnose Helicobacter pylori infection.-Gastroenterology 1995;109:136-141.

15. Douglas J Passaro, E Julia Chosy, Julie Parsonnet.Helicobacter pylori: Consensus and Controversy.Clinical Infectious Diseases 2002;35:298-304

16. Foxal PA., Hu LT., Mobley LH-Use of polymerase chain reaction-amplified Helicobacter pylori ureasi structural genes for differentiation of isolates.J.Clin.Microb 1992;30:739-741

17. G Bode, U Glasaures, Q Song, A Wichelhaus.Characteristics of Helicobacter pylori in the Oral Cavity of Children-. Hepatology 2001; Vol.49, Supp:a33..

18. Gerhard Treiber, Joachim Witting, Susanne Ammon, et al-. Clinical Outcome and Influencing Factors of a New Short-term Quadruple Therapy for Helicobacter pylori Eradication.Arch Intern Med 2002;.162:

19. Goodwin CS, Armstrong JA- Microbiological aspects of Helicobacter pylori-Eur J Clin Microbiol 1990; 9:1-13

20. Grino P. Pascual S, Such J, Casellas AJ. Et alComparison of diagnostic methods for Helicobacter pylori infection in patients with upper gastrointestinal bleeding-Scand J Gastroenterol 2001;36(12):1254-8

21. Grove DI, McLeay RA, Byron KE, Koutsouridis G.- 
Isolation of Helicobacter pylori after transport from a regional laboratory of gastric biopsy specimens in saline, Portagerm pylori or cultured on chocolate agar-.Pathology 2001; 33:362-4

22. Hazel Mitchell and Francis Mègraudt.-Epidemiology and diagnosis of Helicobacter pylori infection.Helicobacter 2002; volume 7 Supplement1

23. Ikue Taneike, Yukiko Tamura, Toshiaki Shimizu, Yuichiro Yamashiro, Tatuo Yamamoto-Helicobacter pylori Intrafamiliar Infections: Change in Source of Infection of a Child from Father to Mother after Eradication Therapy-Clinical and Diagnostic Laboratory Immunology, 2001;.8(.4):731-739

24. Yakoob J, Hu G, Fan X, Zhang Z.-Helicobacter pylori detection in Chinese subjects: a comparison of two common DNA fingerprinting methods.-Br J Biomed Sci 2001;58(4):239-43

25. James Versalovic ,James G.Fox- Helicobacter In Murray PR,ed. Manual of Clinical Microbiology, 7th edn, sect IV, 716-726. 1999

26. J Y H Lee, HM Windsor, BJ Marshall- Helicobacter pylori Trasmission between Spouses in Western Australia - An International Journal of Gastroenterology and Hepatology, 2001; Vol 49, suppl.I:, A31

27. John Holton- Clinical relevance of culture: why, how, and when-Helicobacter 1997; volume 2 Supplement1

28. Kisa O, Albay A, Mas MR, Celasun B, Dogancy LThe evalutation of diagnostic methods for the detection of Helicobacter pylori in gastric biopsy specimens-Diagn Microbiol Infect Dis 2002; 43(4):251-5

29. K LM McColl, LS Murray, D Gillen, A Walker, A Wirz, et al.- Randomised trial of endoscopy with testing for Helicobacter pylori compared with non-invasive $\mathrm{H}$ pylory testing alone in the management of dyspepsia-BMJ 2002;324(7344):999

30. Lind T, Valdhuyzen van Zanten S, Unge P et al.Eradication of Helicobacter pylori using one-week triple therapies combining omeprazole with two antimicrobial: the Mach 1 study- Helicobacter 1996;1(3):138-44

31. Malfertheiner P, Megraud F, O'Morain C et al.Current concepts in the management of Helicobacter pylori infection- the Maastricht 2-2000 Consensus Report.-Aliment Pharmacol Ther 2002;16(2):167-80

32. Marshall BJ.- Unidentified curved bacillus on gastric epithelium in active chronic gastritis-Lancet. 1983;1: 1273-1275.

33. Marshall BJ, Warren JR, Francis GJ.-Rapid urease test in the management of Campylobacter pyloridis associated gastritis-Am J Gastroenterol 1987;82:200-210.

34. Masahiro Asaka, Kiichi Satoh, Kentaro Sugano et al.Guidelines in the Management of Helicobacter pylori infection in Japan-Helicobacter 2001; 6 (3)

35. Megraud F.-Resistance of Helicobacter pylori to antibiotics: the main limitation of current protonpump inhibitor triple therapy-Eur J Gastroenterol Hepatol $1999 ; 11$ Suppl2:s35-7;s43-5

36. Mendez GL, Burns BP, Hazell SL. Characterisation of glucose transport in Helicobacter pylori Biochim Biophiys Acta 1244:269-276,1995

37. National Institutes of Health Consensus ConferenceHelicobacter pylori in peptic ulcer disease-JAMA 1994;272:65-69

38. Owen RJ, Desai M, FIgura N, Bayeli PF, Di Gregorio L, Russi M, Musmanno RA.-Comparison between degree of histological gastritis and DNA fingerprints, Cytotoxicity and adhesivity of Helicobacter pylori from different gasrtric sites-Eur J Epidemiol.
1993;9(3):315-321

39. Perez-Trallero, E., M. Montes, M. Alcorta, P. Zubillaga, E. Telleria-.Non endoscopic method to obtain Helicobacter pylori for culture-Lancet 1995;345:622-623

40. Phadnis SH, Parlow MH, Levy M., et al.-Surface localizazion of Helicobacter pylori urease and a heart shock protein homolog requires bacterial autolysis.Infect Immun 1996;64:905-912

41. Piccolomini R, Di Bonaventura G, Festi D, et al.Optimal combination of media for primary isolation of Helicobacter pylori from gastric biopsy specimens.J Clin Microb 1997;35:1541-1544

42. PL Melito, C Munro, PR Chipman, DL Woodward, TF Booth, FG Rodgers-Helicobacter winghamensis sp. Nov., a novel Helicobacter sp. Isolated from Patients with Gastroenteritis-J Clin Microb 2001;39(7):2412-2417

43. R Piccolomini, G di Bonaventura, B DainelliMicrobiologia Clinica di Helicobacter pylori-GIMMOC 1999; vol. III N $\mathrm{N}^{\circ} 3$

44. Robert P H Logan, Marjorie M Walker-Epidemiology and diagnosis of Helicobacter pylori infection.-BMJ 2001;323:920-922

45. Sachs G, Weeks DL, Melchers K, Scott DR-The Gastric Biology of Helicobacter pylori Annu Rev Physiol $2002 ; 2$

46. Samuels AL, HM Windsor, GY Ho, LD Goodwin, B J Marshall.-Culture of Helicobacter pylori from a gastric string may be an alternative to endoscopic biopsy.- J Clin Microb 2000;38: 2438-2439

47. Shan-Rui Han, Hans-Cristoph E Zschausch, HeinzGeorg W Meyer, et al.-Helicobacter pylori; Clonal Population Structure and Restricted Transmission within Families Revealed by Molecular Typing.-J Clin Microb 2000,.38(10):3646-3651

48. Sebastian Suerbaum, and Pierre Michetti.Helicobacter pylori infecion-.N Engl J Med. 2002;347(15):1175-86

49. Spiegelhalder C, Gerstenecker B, Kersten A, Schiltz E, Kist M-.Purification of Helicobacter pylori superoxide dismutase and cloning and sequencing of the gene-.Infect Immun 1993;61:5315-5325

50. T Alarcon, D Domingo, M Lopez-Brea-Effect of Difference Conditions in the Growth of Helicobacter pylori in Liquid Media.-International Journal of Gastroenterology and Hepatology,2001; 49,Suppl. II:,46.

51. Tomb JF, White O, Kerlavage AR et al.- The complete genome sequence of the gastric pathogen Helicobacter pylori.-Nature1997; 388:539-547

52. Treiber G, Malfertheiner P-.Helicobacter pylori 2002-Schweiz Rundsch Med Prax 2002;91(18):773-8

53. Yakoob J, Hu G, Fan X, Zhang Z.-Helicobacter pylori detection in Chinese subjects: a comparison of two common DNA fingerprinting methods-.Br J Biomed Sci 2001;58(4):239-43

54. Zhukhovitsky VG, Didenko LV,Konstantinova ND,Forms with defected cell wall of Helicobacter pylori in vitro-Abstract (A7)Eur.Congress.Atene 2002 